\title{
FORMAR A LOS ESTUDIANTES EN EL MÉTODO EXPERIMENTAL: ¿UTOPÍA O PROBLEMA SUPERADO?
}

DUMON, A.

GREDIC - U. F, R. Sciences et Techniques. Université de Pau. Avenue de l'Université. 64000 PAU (France).

\section{SUMMARY}

A laboratory work teaching sequence, based on the practical work objectives definition, on the progressive development of autonomy, and a hierarchized learning task is experimented. The evaluation of students results show that the learning strategies lead to a real mastery of experimental process.

\section{PLANTEAMIENTO DEL PROBLEMA}

Si hacemos caso de las instrucciones oficiales francesas (MEN 1985, 1987), así como de los escritos de algunos autores (Astolfi 1978, Giordan 1978, Gólmez 1978, Helligman 1982, Host 1982, Kempa 1977 y 1986, Leclercq 1980, Swain 1974, Tompson 1971, UNESCO 1981), el desarrollo de las capacidades relacionadas con el método científico es la meta principal de las actividades experimentales de la enseñanza secundaria. EI trabajo de laboratorio debe hacer que los alumnos sean capaces de:

1) identificar el problema, plantearse cuestiones y tener ganas de contestarlas por sí mismos,

2) formular hipótesis,

3) imaginar comprobaciones experimentales de las hipótesis,

4) poner en tela de juicio sus representaciones (o hipótesis) iniciales a partir del experimento,

5) buscar la información necesaria para la resolución del problema,

6) resolver el problema ideando experimentos,

7) interpretar resultados y fenómenos observados,

8) imaginar aplicaciones y extrapolaciones de los "descubrimientos" que se han hecho.

Puede parecer pues que, a nivel universitario, nuestro plantearmiento inicial no tenga razón de ser. Sin embar- go, se deduce de un estudio bibliográfico (Dumon 1986) que muchos autores mencionan algunos fines y objetivos que conciernen al aprendizaje de capacidades metodológicas como fines y objetivos que hay que desarrollar prioritariamente en las prácticas de química en la Universidad (Hanson 1982, Johnstone 1979, Ophardt 1979, Pickering 1987, Silberman 1987, Singer 1984, Warren 1987). Resulta pues que no se domina la práctica de un método científico experimental al entrar en el primer ciclo universitario. Esta observación viene confirmada por una encuesta reciente (Roletto 1988), que pone de manifiesto que, en Francia, en la enseñanza secundaria, los trabajos experimentales actuales están orientados prioritariamente hacia la ilustración y la aplicación de conceptos teóricos.

Así pues, ¿es utópico querer alcanzar el objetivo de formar a los estudiantes en el método experimental?

A lo largo de este estudio hemos querido:

- Evaluar el nivel de preparación de los estudiantes que entran en el primer ciclo respecto de este método.

- Concebir una posible estrategia de enseñanza para alcanzar este objetivo.

- Evaluar la eficacia de esta estrategia. 


\section{EVALUACIÓN DE LA PUESTA EN PRÁC- TICA DE UN METODO EXPERIMENTAL AL PRINCIPIO DEL PRIMER CICLO UNIVER- SITARIO}

Este estudio es la continuación de un primer trabajo realizado en Marruecos (Dumon 1988).

Muestra: 50 estudiantes de primer año de Universidad que escogieron la rama de química.

\section{Procedimiento de evaluación:}

- Actividades propuestas: Se evaluó mediante dos problemas basados en los conocimientos previos del conjunto de estudiantes (conceptos de ácido y de base, indicadores de color, proporción de ácido y base) si los estudiantes eran capaces de poner de manifiesto las 7 capacidades referidas en el cuadro I.

\section{Primer problema (extraído de Ophardt 1978)}

"Se dispone de 4 disoluciones A, B, C, D. Asociar a cada una de estas disoluciones la fórmula del compuesto en solución: $\mathrm{HCl}, \mathrm{NaOH}, \mathrm{H}_{2} \mathrm{O}$, fenolftaleína $(\varnothing \emptyset)$. Hay que representar mediante un esquema el proceso sistemático que se debe poner en práctica para resolver el problema.

Consigna: Hay que someter al juicio del profesor toda idea, propuesta o petición de ayuda antes de realizarla".

Nota: El estudiante disponía de disoluciones conocidas para comprobar sus hipótesis.

\section{Segundo problema (sacado de Johnstone 1979)}

"Hay que determinar la concentración en ácido cítrico de tres bebidas de frutas (zumo de limón, zumo de naranja, jarabe de grosella negra) mediante una valoración volumétrica de una solución de sosa molar. Hay que proponer un plan de experimentación. Para ello, se debe:

- Buscar información complementaria. ¿Cuál?

- Plantearse cierto número de preguntas previas y contestarlas uno mismo.

\section{Consigna: La misma que para el primer problema.}

- Recogida de información. La evaluación se realizó a partir de los datos, tomados por escrito, de todas las interacciones estudiantes-profesor (ya que las interacciones estudiante-estudiante estaban prohibidas) y de la observación del comportamiento de los estudiantes.

\section{Resultados}

Se anotaron en el cuadro I los porcentajes de estudiantes que pusieron de manifiesto las capacidades especificadas $(+)$, que las pusieron de manifiesto de manera incompleta o tras una ayuda $( \pm)$, y que no las pusieron de manifiesto $(-)$.

\section{Cuadro I}

Proporciones de estudiantes que demostraron las capacidades relacionadas con el método experimental.

\begin{tabular}{|c|c|c|c|c|c|c|}
\hline Micdulo & \multicolumn{3}{|c|}{ if problemo } & \multicolumn{3}{|c|}{$2^{2}$ problema } \\
\hline Copocidodes & + & \pm & - & + & \pm & - \\
\hline $\begin{array}{l}\text { 1. Idenlificar el problema } \\
\text { Buscor la información } \\
\text { pertlirente }\end{array}$ & 82 & 16 & 2 & 31 & 37 & 32 \\
\hline $\begin{array}{l}\text { 2. Emisis hipóresis } \\
\text { Plontearse preguntos }\end{array}$ & 27 & 4 & 69 & 6 & 10 & 84 \\
\hline $\begin{array}{l}\text { 3. Experimentalmenle: } \\
\text { Comprobor los hipólesis } \\
\text { o corlestar a las } \\
\text { preguntos }\end{array}$ & $\frac{2}{40}$ & $\frac{1}{0}$ & 54 & - & - & 100 \\
\hline $\begin{array}{l}\text { 4. Poner en telo de juicio } \\
\text { sus hipótesis }\end{array}$ & 20 & 7 & 73 & 29 & 2 & 69 \\
\hline $\begin{array}{l}\text { 5. Proponer un plan } \\
\text { experimenial; maleriol } \\
\text { que hoy que emplear }\end{array}$ & 24 & - & 76 & 18 & 29 & 53 \\
\hline $\begin{array}{l}\text { o. Resolver el problema } \\
\text { medianle un método } \\
\text { adecuodo }\end{array}$ & 24 & 27 & 49 & & objeto & \\
\hline $\begin{array}{l}\text { 7. Representar } \\
\text { esquemálicamente el } \\
\text { métado empleado } \\
\text { Redactar un modo } \\
\text { operatorio adecuado }\end{array}$ & 0 & 0 & 100 & 12 & 18 & 70 \\
\hline
\end{tabular}

\section{Comentarios}

Capacidad 1. Casi todos los estudiantes se dieron cuenta de que el primer problema consistía en la identificación de un ácido y de una base mediante un indicador. En cambio, sólo el $31 \%$ de los estudiantes consiguió identifficar el segundo problema, que radicaba en la búsqueda de información pertinente para su resolución (fórmula del ácido cítrico, fuerza del ácido, aspecto de la curva de valoración, lista de los indicadores de color y zonas de viraje).

Capacidad 2. Antes de empezar a resolver el primer problema, sólo el $27 \%$ de los estudiantes formuló al menos una hipótesis sobre el cambio de color del indicador según el medio: "si $\emptyset \emptyset$ se vuelve... entonces tenemos...". Los demás empezaron a "manipular" sin reflexión previa.

Para construir el plan experimental del segundo problema, el estudiante tenía que plantearse ciertas preguntas como:

- ¿Cambiará de color el zumo con el pH?

- ¿Será visible el cambio de color del indicador? Si no, ¿cómo habrá que proceder?

- ¿Es adecuada la concentración propuesta de la solución de sosa?

- ¿Qué equipo hay que emplear para la valoración? 
Se observa que muy pocos estudiantes $(6 \%)$ se plantean preguntas antes de actuar.

Capacidad 3. En lo concerniente al primer problema, el $40 \%$ de los estudiantes que emitieron una hipótesis se plantearon comprobarla mediante las disoluciones conocidas que tenían a su disposición.

En cambio, no se observó ningún intento de contestar, a partir del experimento, alguna pregunta del segundo problema.

Capacidad 4. EI $20 \%$ de los estudiantes que emitió alguna hipótesis para el primer problema no pusieron de manifiesto la capacidad 3 , pero demostraron que estaban dispuestos a replantear su idea inicial.

En lo que se refiere al segundo problema, sólo después del experimento les pareció necesario replantearse la concentración de la sosa al $29 \%$ de los estudiantes (el $52 \%$ realizó la valoración).

Capacidad 5. El 24\% de los estudiantes propuso correctamente, antes de ponerse a "manipular", un plan experimental que permitiera diferenciar las soluciones (primer problema); y el $18 \%$ escogió el material adecuado para realizar la valoración del ấcido cítrico.

Capacidad 6. El $24 \%$ de estudiantes que propuso un plan experimental (primer problema) consiguió identificar las 4 soluciones. Otros estudiantes $(27 \%)$ consigueron resolver el problema mediante un proceso sistemático basado en la observación y la comparación de los resultados obtenidos durante la mezcla de las disoluciones desconocidas con mezclas testigo realizadas a partir de disoluciones conocidas.

Capacidad 7. Ningún estudiante fue capaz de sintetizar mediante un esquema el proceso que siguió para identificar las 4 soluciones del primer problema, y sólo el $12 \%$ redactó un procedimiento correcto para la valoración de ácido cítrico.

\section{Conclusión}

Si e1 24\% de los estudiantes empleó un proceso razonado que se podía considerar científico para resolver un problema relativamente sencillo, sólo el $12 \%$ consiguió proponer un plan experimental correcto para resolver un problema más complejo. Se pueđe concluir, pues, de acuerdo con nuestro estudio precedente (Dumon 1988), que el proceso experimental sólo aparece en estado embrionario en los estudiantes que entran en primer año de primer ciclo universitario.

\section{CONCEPCIÓN DE UNA ESTRATEGIA DE ENSENANZA}

La concepción de la secuencia (7 sesiones de $4 \mathrm{~h}$ ) de clases experimentales se basa en:
- un estudio bibliográfico previo (Dumon 1986, 1987 y 1988),

- el análisis de las expectativas de los profesores y los estudiantes (Dumon 1989, 1990),

-experiencias anteriores (Dumon 1987, Bonnecaze 1984).

Se escogieron entonces:

- los objetivos

- la estrategia de enseñanza

- el procedimiento de evaluación de los estudiantes.

\section{Objetivos}

El objetivo final que fija la orientación de la enseñanza experimental es: «el estudiante debe ser capaz de utilizar un método científico para la resolución de un problema.»

Escogimos este objetivo siguiendo el punto de vista según el cual la enseñanza experimental, en el primer año de primer ciclo, puede justificarse por sí sola por las aptitudes relacionadas con el método experimental que se supone debe desarrollar (Dumon 1986). Esta elección viene reforzada por algunos estudios (Johnstone 1979, Hearle 1973) que muestran que el hecho de querer hacer asimilar nociones teóricas mediante trabajos experimentales conduce a resultados poco concluyentes cuando los estudiantes tienen que aprender a manipular al mismo tiempo.

Este objetivo se concreta por objetivos intermedios y objetivos propios de cada manipulación que se comunican a los estudiantes (Dumon 1987 y Anexo I).

\section{Estrategia de enseñanza}

La puesta en práctica de la secuencia de enseñanza resultó, no sólo de:

- Ia orientación definida por el objetivo final,

- tener en cuenta las normas institucionales (organización general de las enseñanzas, medios materiales y financieros, aula(s) disponible(s),

- análisis del público estudiante (aptitudes, motivación, nivel de desarrollo intelectual),

sino también de consideraciones de tipo pedagógico.

Nuestro objetivo final corresponde al nivel superior de la taxonomía de las operaciones cognitivas de D'Hainaut (1983): la resolución de problemas. Es decir, que, ante una tarea con la que nunca se ha enfrentado anteriormente, el estudiante debe dar una respuesta a partir de la combinación (no aprendida) de reglas, procedimientos y principios aprendidos.

Es necesario, pues, estructurar en el tiempo los aprendizajes para hacer que aquél domine progresivamente las otras categorías de operaciones cognitivas que le permitirán alcanzar este nivel.

Durante su trabajo en el laboratorio, el estudiante está sometido a distintas influencias (Johnstone 1979): 
- las instrucciones escritas (manual o fichero),

- las instrucciones orales,

- el nombre de los aparatos y de los productos que ha de reconocer y asociar,

- Ias habilidades manipulatorias nuevas que ha de aprender,

- la teoría que tiene que recordar,

- el principio de funcionamiento de los aparatos que ha de aprender o recordar,

- la información originada por la experiencia.

Estas influencias llevan al estudiante a un estado de sobrecarga inestable del que tiene tendencia a salir:

- siguiendo paso por paso el modo operatorio, sin intentar entender el sentido de su trabajo,

- concentrándose en una parte y excluyendo el resto,

- copiando la acción de los demás,

- etc.

No consigue distinguir lo esencial de lo que es accesorio $y$, entonces, parece ilusorio querer enseñarle algo. Nos hemos esforzado por remediar estas perturbaciones:

- indicando claramente al estudiante adónde se dirige (los objetivos),

- formulando los textos de los experimentos en función de los objetivos que hay que alcanzar (evitar las recetas), - distinguiendo en el documento escrito: lo que es preparatorio del experimento, to que es esencial, lo que es accesorio,

- asegurándose de que el estudiante ya domina los objetivos de tipo manipulativo antes de utilizarlos para alcanzar objetivos de interpretación.

La estrategia de enseñanza que ponemos en práctica es modular (Dumon 1988). El conjunto de estudiantes aborda simultáneamente cada módulo en una misma sesión.

Se ha estructurado en el tiempo la sucesión de los distintos módulos (Anexo 1) y su nivel de dificultad de manera que el estudiante:

- aplique $\rightarrow$ justifique $\rightarrow$ modifique un modo operatorio,

- seleccione a continuación el equipo/método/ productos (conocidos) para resolver un problema con el que no se ha enfrentado nunca anteriormente,

- proponga finalmente un equipo / método / productos (no necesariamente conocidos) para resolver un problema (đesconocido para él).

Para alcanzar los objetivos propios de cada módulo, el estudiante dispone de un documento-guía que le indica que debe: preparar las manipulaciones con la ayuda de un manual preestablecido, buscar respuestas a ciertas preguntas planteadas en el texto acerca de las manipulaciones, justificar cada acción que se ha llevado a cabo respondiendo a Ias preguntas propuestas, cooperar en parejas para poner en comín resultados o buscar un protocolo experimental. Además, se introdujo en el aula de prácticas un microordenador como instrumento didáctico para favorecer ciertos aprendizajes (Bonnecaze 1984).
El estudiante que emplea un proceso experimental para resolver un problema debe tener iniciativas y autonomía. Pero, teniendo en cuenta sus aprendizajes anteriores, estas actitudes no aparecen espontáneamente. Hemos procurado hacer evolucionar el grado de autonomía del estudiante (de una autonomía restringuida hacia una autonomía verdadera) pasando progresivamente, durante la evolución del aprendizaje, de la simple relación Profesor $\rightarrow$ Estudiante, a la relación Profesor $<->$ Estudiante(s) $<\rightarrow$ Estudiante(s).

Entonces, el trabajo del profesor debe ser animar la sesión:

- incitando a un diálogo real, a la confrontación de ideas, a participar en su aprendizaje al máximo de estudiantes, - reconociendo el derecho al error, estimulando la confianza en ellos mismos.

\section{Procedimiento de evaluación de resultados}

Hemos descrito este procedimiento en un trabajo anterior (Dumon 1987). Recordemos simplemente que:

- es de tipo formativo,

- la recogida de información para la evaluación se realiza, para cada manipulación, mediante una tabla de especificaciones en donde figuran las capacidades evaluadas (capacidades identificadas en un marco de referencia más general),

- los procedimientos de recogida de información son múltiples: pruebas escritas, preguntas orales, observación de comportamientos, comunicación escrita de los resultados,

- se evalúa el haber alcanzado o no el objetivo final en un examen final durante el cual el estudiante debe resolver un problema experimental nuevo (presentado en 10 líneas como máximo), pero cuyo tema general esté relacionado con los módulos que se han tratado durante el curso.

\section{EVALUACIÓN DE LA EFICACIA DE LA ESTRATEGIA DE APRENDIZAJE}

Esta evaluación se basará en:

- el test inicial,

- la evolución en el dominio de las capacidades durante los aprendizajes,

- Ia evaluación de la competencia en el examen final.

\section{Evolución de la competencia de las capacidades}

Se reagrupa el conjunto de observaciones realizadas en 10 capacidades (Cuadro II). Se ha determinado para cada módulo, a medida que se llevaban a cabo las observaciones, el porcentaje de estudiantes que demostraron el dominio de las capacidades ( $t$ ), que lo hicieron parcialmente $( \pm)$, o no lo hicieron en absoluto (-). 
Cuadro II

Evolución del dominio de las capacidades a través de los módulos.

\begin{tabular}{|c|c|c|c|c|c|c|c|c|c|c|c|c|c|c|c|c|c|c|c|c|c|}
\hline Módulo & \multicolumn{3}{|c|}{$\mathbf{I}$} & \multicolumn{3}{|c|}{2} & \multicolumn{3}{|c|}{3} & \multicolumn{3}{|c|}{4} & \multicolumn{3}{|c|}{5} & \multicolumn{3}{|c|}{0.7} & \multicolumn{3}{|c|}{ FINAL } \\
\hline Capocidodes & - & \pm & + & - & \pm & + & - & \pm & + & - & \pm & + & - & \pm & + & - & \pm & + & - & \pm & + \\
\hline $\begin{array}{l}\text { 1. Dominio de los nociones } \\
\text { prerequeridas tcomprensión, } \\
\text { oplicaciónl. }\end{array}$ & 53 & 29.5 & 17,5 & 27 & 14 & so & 17 & 22 & ol & 20 & 17 & 57 & 0 & 25 & 75 & & & & 9 & 13 & 78 \\
\hline $\begin{array}{l}\text { 2. Eúsqueda de la información } \\
\text { pertinente / percepción de } \\
\text { las componientes del problema }\end{array}$ & & & & & & & 50 & 0 & so & 20 & 0 & 80 & & & & & & & 10 & 31 & 53 \\
\hline 3. Habilidades manipulotorias & 50 & 25 & 25 & 30 & $3 \Delta$ & 36 & 12.5 & 12,5 & 75 & & & & & & & & & & 0 & 28 & 72 \\
\hline 4. Calidad de los medidas & 0 & 83 & 17 & 5 & 18 & 77 & & & & & & & & & & & & & & & \\
\hline $\begin{array}{l}\text { 5. Seguimienlo de un modo } \\
\text { operotivo }\end{array}$ & 59 & 18 & 23 & 0 & 9 & 91 & & & & & & & & & & & & & & & \\
\hline $\begin{array}{l}\text { 6. Propuesto de un modo } \\
\text { operalivo }\end{array}$ & 14 & 57 & 29 & & & & 22 & 32 & 40 & is & 20 & 58 & 0 & 29 & 71 & & & & 9 & 42 & 49 \\
\hline $\begin{array}{l}\text { 7. Conlrol de la validez de los } \\
\text { resultodos }\end{array}$ & 90 & 10 & 0 & & & & 37,5 & 25 & 37,5 & & & & 12.5 & 12.5 & 75 & & & & 53 & 13 & 34 \\
\hline $\begin{array}{l}\text { 8. Análısis crílico de } \\
\text { los resuliodos }\end{array}$ & & & & & & & 50 & - & 44 & & & & & & & & & & 33 & 47 & 20 \\
\hline $\begin{array}{l}\text { 9. Resolución del problento } \\
\text { ten ei sentido de d'Hainaull] }\end{array}$ & & & & & & & & & & & & & & & & 2 & 35 & 63 & 20 & 27 & 47 \\
\hline 10. Proceso experimentol & & & & & & & & & & & & & & & & & & & 18 & 22 & $\Delta 0$ \\
\hline
\end{tabular}

Se puede observar que la competencia de las capacidades progresa adecuadamente durante la secuencia de enseñanza. Debemos tener en cuenta dos cuestiones:

- La evaluación formativa llevada a cabo durante los módulos concierne a la pareja y no a un estudiante en particular. Además esta evaluación se realiza cuando las relaciones del estudiante con su entorno (profesores y estudiantes) son intensas. No se le puede pues, atribuir, más que un carácter indicativo.

- En la organización de la enseñanza, conviene diferenciar la serie de módulos 1 a 5 de los dos últimos. La primera serie está concebida de manera que el estudiante, mediante la enseñanza de diferentes métodos de valoración, Ilegue a proponer un procedimiento (de tipo conocido) para realizar la valoración de un producto (nuevo). Los dos últimos módulos deben permitir que el estudiante identifique las variables que intervienen en un fenómeno y luego las ponga en relación. El método de trabajo que hemos adoptado aquí se basa en el hecho de poner en común los resultados y la discusión en grupo, por lo que es difícil tlevar a cabo la evaluación individual de las distintas capacidades.

Parece, pues, que una proporción importante de estudiantes haya alcanzado los objetivos fijados para la secuencia de enseñanza.

\section{Evaluación de la competencia durante el examen final}

En este caso, sólo se evalúan las aptitudes propias de cada estudiante.

Se puede decir que durante una actividad de resolución de problemas (puesta en práctica de procesos que no han visto anteriormente, pero que resultan de la combinación de operaciónes conocidas, para resolver un problema nuevo):

- todos muestran habilidades manipulativas correctas, - el $91 \%$ domina bastante bien las nociones prerequeridas y son capaces de proponer un procedimiento adecuado,

- el $84 \%$ efectúan adecuadamente la búsqueda de información pertinente,

- el $82 \%$ pone en práctica un proceso al que se puede calificar de experimental,

- el $74 \%$ consigue Ilegar a la solución del problema bastante satisfactoriamente,

- al $67 \%$ se le ocurre analizar su(s) resultado(s) teniendo en cuenta las incertidumbres, pero sólo el $20 \%$ lo hace correctamente,

- el $47 \%$ controla ta validez de su(s) resultado(s), pero sólo el $34 \%$ to hace espontáneamente. 
Es preciso hacer una observación. Si los estudiantes consiguen poner en práctica un proceso que les permita resolver el problema, parece que la capacidad de analizar de manera crítica los tesultados obtenidos, puesta de manifiesto durante las actividades dirigidas, no constituye una de sus mayores preocupaciones cuando su trabajo es totalmente autónomo. Su atención parece fijarse entonces en la obtención de un resultado sin plantearse preguntas acerca de su validez. Este comportamiento puede explicarse por el hecho de que el examen será puntuado y se efectúa en un tiempo limitado, lo que comesponde a una situación nueva para ellos, que les perturba: la competencia, pues, no parece adquirida.

\section{Conclusión sobre la eficacia de la estrategia de apren- dizaje}

Respecto del test inicial al que sometimos a nuestra población estudiantil, podemos concluir que las actividades propuestas y su organización conducen en efecto a una progresión en los aprendizajes y a un cierto dominio del "proceso" experimental. Pero se plantean dos preguntas:

- ¿No sería posible obtener resultados idénticos, tal vez incluso mejores, a partir de un enfoque distinto? El tamaño reducido de nuestra población no nos permitió

\section{NOTA}

Este artículo ha sido traducido del original francés. comparar las competencias de dos muestras sometidas a métodos diferentes.

- ¿Se transferirán los logros que consiguieron los estudiantes al final de la secuencia de enseñanzas a estudios posteriores?: ¿pasarán a ser competencias?

\section{CONCLUSIÓN}

No pretendemos haber elaborado el método de enseñanza ideal; se pueden adoptar otros procesos, optar por otras elecciones. Somos conscientes de las imperfecciones del procedimiento de evaluación de la estrategia que hemos adoptado. Sin embargo, pensamos haber alcanzado los dos fines que habíamos fijado para este trabajo.

- Evaluar el nivel de preparación de los estudiantes respecto del método experimental al inicio del primer ciclo universitario: como ya habíamos apuntado (Dumon 1987) pocos estudiantes demuestran tener aptitudes para este tipo de método.

- Ilustrar cómo, mediante la elaboración de una estrategia de enseñanza pensada y organizada, es posible hacer que nuestros estudiantes adquieran un comportamiento un poco científico. Parece que lo conseguimos.

\section{ANEXO I}

\section{TÍTULOS Y OBJETIVOS FINALES DE LOS MÓDULOS}

Módulo l. Introducción al análisis cuantitativo

Después de tener conocimiento del utillaje volumétrico y haber aprendido a manipularlo, el estudiante debe ser capaz de:

- llevar a cabo las operaciones elementales de volumetría escogiendo el material correspondiente a la precisión exigida, - tener unat actitud crítica respecto de los resultados experimentales (incertidumbre, etrores sistemáticos, reproductividad, validez, honestidad).

Módulo 2. Introducción a las reacciones ácido-base en solución acuosa

El estudiante debe ser capaz de valorar un ácido con una hase fuerte, mediante un $\mathrm{pH}$-metro o un indicador de color, con una precisión superior a 3\% (siguiendo un modo operativo dado).
Módulo 3. Estudio cuantitativo de reacciones ácido-base en solución acuosa

El estudiante debe ser capaz de:

- preparar una solución de concentración dada (ácida o básica) a partir de un producto comercial,

- determinar la concentración exacta de una solución ácida mediante una valoración volumétrica o potenciométrica (justificar y luego proponer los modos operatorios).

Modulo 4. Introducción al análisis volumétrico de un cuerpo que libera a otro

El estudiante debe ser capaz de proponer un plan experimental para llevar a cabo una valoración empleando una reacción ácido-base o de óxido-reducción después de haber: 
- buscado la información necesaria entre la que se halla en el manual (incluyendo las manipulaciones anteriores),

- contestado por medio de experimentos algunas preguntas previas.

Modulo S. Estudio a partir de un compuesto de funciones múltiples $\mathrm{K}_{2} \mathrm{CrO}_{4}$

A partir de información sacada del manual preestablecido, deben ser capaces, elaborando los procedimientos, de llevar a cabo la valoración de un compuesto susceptible de disociarse en solucion, interviniendo sobre las distintas propiedades de los iones obtenidos, y realizar un estudio comparativo de los resultados.

\section{REFERENCIAS BIBLIOGRÁFICAS}

ASTOLFI, J.P., GIORDAN, A., GOHAN, G., HOST, V., MARTINAND, J.L., RHUMELHARD, G. y ZADOUNAIISKY, G., 1978. Quelle éducation scientifique pour quelle société? (PUF: París).

BONNECAZE, G., CARDY, H., IRATCABAL, P y DUMON, A., 1984. Actualité Chimique. Abril, pp. 38-40.

D'HAINAULT, L., 1983. Des fins aux objectifs de l'éducation. (Nathan-Labor: Bruselas).

DUMON, A., 1986. Actualité Chimique. Noviembre, pp. $57-$ 66.

DUMON, A., 1986. Revue des Sciences de l'Éducation, Vol. 13, pp. $421-435$.

DUMON, A., 1987. Actualité Chimique. Marzo, pp. 81-85.

DUMON, A., 1988. Revue Française de Pédagogie, Vol. 84 , pp. 29-38.

DUMON, A., 1989. Actualité Chimique. Enero, pp. 28-31.

DUMON, A., 1990. Soumis pour publication à Bulletin du Centre International Francophone pour l'Education en Chimie.

DUMON, A., ABTAL, E., 1987. Pédagogiques, Vol. 7, pp. 77 96.

DUMON, A., ABOU-SALEH, J. y ABTAL, E., 1988. Bulletin du Centre International Francophone pour l'Education en Chimie, Vol. 2, pp. 31-36.

DUMON, A. y PICKERING, M., 1990. Journal of Chemical Education, Vol. 67, pp. 959-960.

GIORDAN, A., 1978. Une pédagogie pour les sciences expérimentales. (Le Centurion Formation: París).

GOLMEZ,F., DELACOTE, G. y RICHARD, J.E., 1978. Revue Française de Pédagogie, Vol. 45, pp. 55-65.

Guide pour les professeurs de sciences. (Les presses de I'UNESCO: París).

HANSON, A.L., 1982.Journal of Chemical Education, Vol. 59, p. 671 .

HEARLE, R.J., 1973. Ph. D. dissertation, Untversity of Maryland.

\section{Módulo 6-7. Criometría}

El estudiante debe ser capaz de utilizar un proceso científico experimental para resolver un problema nuevo. Para ello, deberá:

- tener iniciativa, es decir, utilizar procesos (y conocimientos) adquiridos durante las manipulaciones anteriores,

- identificar las variables que intervienen en el fenómeno físico-químico que deben estudiar y relacionarlas entre ellas para deducir una ley.

HELLIGMAN, C., 1982. European Journal of Science Education, Vol. 4, pp. 29-43.

HOST, V., 1982. En Martinand, J.L., 1982. Actes des 4èmes journées sur l'Éducation sicentifique, Chamonix, 4-6 février.

JOHNSTONE, A.H. y WHAM, A.J.B., 1979. Education in Chemistry, vol. 16, pp. 16-17.

KEMPA, R., 1977. Physics Education, Vol. 12, pp. 364-367.

KEMPA, R., 1986. Assessment in Science. (Cambridge University Press: Cambridge).

LECLERCQ, D., 1980. Construire et utiliser des séquences d'entraîment à la résolution de problèmes, document du laboratoire de pédagogie expérimentale. (Université de Liège).

Ministère de l'Éducation Nationale, 1985. Collèges - Programmes et lnstructions. (Publication du C.N.D.P.).

Ministère de l'Éducation Nationale, 1987. Sciences Physiques dans les terminales scientifiques - Objectifs et Procédures d'évaluation. (Direction des Lycées et Collèges).

OPHARDT, C.E., 1978. Journal of Chemical Education, Vol. 55, pp. 485-488.

PICKERING, M., 1987. Journal of Chemical Education, Vol. 64 , pp. $521-529$.

ROLETTO, E., CARRETO, J. y VIOVY, R., 1988. Bulletin du Centre International Francophone pourl'Éducation en Chimie, Vol. 3, pp. 27-42.

SILBERMAN, R., DAY, S., JEFFERS, P., KLANDERMAN, K., PHILLIPS, G. y ZIPP, A., 1987. Journal of Chemical Education, Vol. 64, pp. 622-623.

SINGER, B.W. y LOCK, R.J., 1984. Education in Chemistry, Marzo, pp. $51-53$.

SWAIN, J.R.L., 1974. Education in Chemistry, Vol. 11, pp. 152-154.

THOMPSON, J.J., 1971. Education in Chemistry, Vol. 8, pp. 170-171, UNESCO 1981.

WARREN, S.W. y PICKERING, M., 1987. Journal of Chemical Education, Vol. 64, pp. 68-69. 